\title{
COMPUTER SEARCH FOR CURVES WITH MANY POINTS AMONG ABELIAN COVERS OF GENUS 2 CURVES
}

\author{
KARL RÖKAEUS
}

\begin{abstract}
Using class field theory one associates to each curve $C$ over a finite field, and each subgroup $G$ of its divisor class group, unramified abelian covers of $C$ whose genus is determined by the index of $G$. By listing class groups of curves of small genus one may get examples of curves with many points; we do this for all curves of genus 2 over the fields of cardinality 5,7,9,11,13 and 16, giving new entries for the tables of curves with many points $[6$.
\end{abstract}

\section{BACKGROUND}

Let $q$ be a prime power and let $g$ be a non-negative integer. We are interested in how many rational points there can be on a projective, smooth and geometrically irreducible curve of genus $g$, defined over the finite field $\mathbf{F}_{q}$; equivalently, how many rational places there can be in a global function field of genus $g$ with full constant field $\mathbf{F}_{q}$. Denote by $\mathrm{N}_{q}(g)$ the maximum possible number of rational places in such a field. The current intervals in which $\mathrm{N}_{q}(g)$ in known to lie for $g \leq 50$, together with references, are given in the tables [6]. In this report we improve upon the lower bounds of these intervals, by constructing curves with many points for some genera over the fields with $q=5,7,9,11,13$ and 16 elements. We do this by going through unramified abelian covers of all genus 2 curves over these fields, using class field theory. The method of looking at such covers has been used before by various authors, see [2]. Still, by doing the systematic search we found some new curves.

The search was done using Magma [1], first in terms of curves, using its arithmetic geometry part; then everything was checked also using its function field part. In this report we have formulated everything in terms of function fields.

Notation. See [15] for an introduction to function fields in one variable. By a function field $F / k$ we will always mean a global function field with full constant field $k$, i.e., $k$ is algebraically closed in $F$. We write $\mathbf{P}_{F}$ for the set of places of $F$ and $\mathrm{Cl}(F)$ for its divisor class group of degree zero divisors.

\section{DESCRIPTION OF THE METHOD}

Class field theory for global function fields: In this section we state the theorems that we use for the construction of the curves; see [11] for details. Let $F / k$ be a global function field. Let $O$ be a rational place of $F$ and set $S=\mathbf{P}_{F} \backslash\{O\}$. We use $F_{O}$ to denote the $S$-Hilbert class field of $F$, i.e., the maximal unramified abelian extension of $F$ (inside a fixed separable closure) in which $O$ splits completely. There is an isomorphism

$$
\psi: \mathrm{Cl}(F) \rightarrow \operatorname{Gal}\left(F_{O} / F\right) .
$$

under which $[P-\operatorname{deg}(P) O]$ maps to $\left[\frac{F_{O} / F}{P}\right]$, the Artin symbol of $P$, for any $P \in \mathbf{P}_{F}$. Fix a subgroup $G$ of $\mathrm{Cl}(F)$ of index $d$ and write $F_{O}^{G}$ for $F_{O}^{\psi(G)}$, the subfield of $F_{O}$ fixed by $\psi(G)$. Then $F_{O}^{G}$ is a Galois extension of $F$ with Galois group isomorphic

Date: June 23, 2011.

${ }^{1}$ The author is supported by The Wenner-Gren Foundations Postdoctoral Grant 
to $\mathrm{Cl}(F) / G$; in particular $\left[F_{O}^{G}: F\right]=d$. A rational place $P \in \mathbf{P}_{F}$ splits completely in $F_{O}^{G}$ if and only if $\left[\frac{F_{O} / F}{P}\right] \in \psi(G)$, i.e., if and only if $[P-O] \in G$.

Therefore, if $F / k$ is a global function field of genus $g$ with rational places $\left\{P_{1}, \ldots, P_{m}\right\}$ and if $G \subset \mathrm{Cl}(F)$ is a subgroup of index $d$ then, for every rational place $O$, we get an unramified extension $F_{O}^{G} / F$ of degree $d$, hence of genus $d(g-1)+1$, with $d \cdot\left|G \cap\left\{\left[P_{i}-O\right]\right\}_{i=1}^{m}\right|$ rational places. Also, $k$ is the full constant field of $F_{O}^{G}$.

Note that, for fixed $G$, two different rational places $O$ and $O^{\prime}$ need not give isomorphic extensions. Although $F_{O}^{G} / F$ and $F_{O^{\prime}}^{G} / F$ have the same genus and isomorphic Galois groups they may have a different number of rational places.

We give an example that can be done by hand, a construction of a genus 6 curve over $\mathbf{F}_{2}$ with 10 rational points.

Example. Let $F$ be the degree 2 extension of $\mathbf{F}_{2}(x)$ given by

$$
y^{2}+x y=x^{5}+x^{3}+x^{2}+x .
$$

It is of genus 2 and has 4 rational places; one containing $x$ and one containing $1 / x$, $P_{0}$ and $P_{\infty}$; and two containing $x+1, P_{1,0}$ and $P_{1,1}$. Moreover, $F$ has two places of degree 2 so its class number is $h=10$. This already gives the existence of four covers of degree 10, namely the $S$-Hilbert class fields $F_{O}$, where $S=\mathbf{P}_{F} \backslash\{O\}$ and $O$ is any rational place in $F$. These are all of genus 11, with 10 rational places.

Let $a=\left[P_{0}-P_{\infty}\right] \in \mathrm{Cl}(F)$. Then $a \neq 0$ (because $F$ is not rational), while $2 a=0$ because $(x)=2 P_{0}-2 P_{\infty}$. Let $G=\{0, a\} \subset \mathrm{Cl}(F)$. If we choose $O=P_{\infty}$ then $\left[P_{\infty}-O\right]=0$ and $\left[P_{0}-O\right]=a$ lie in $G$. The extension $F_{O}^{G} / F$ therefore has degree $10 / 2=5$. It has genus 6 and contains $2 \cdot 5=10$ rational places.

Similarly, if we choose $O=P_{0}$ we also get a genus 6 extension with 10 rational places. However, if we instead choose $O$ to be $P_{1,0}$ or $P_{1,1}$ then $F_{O}^{G}$ is a genus 6 extension with only 5 rational places.

Organization of the search. Let $k$ be a finite field and let $g>1$ be an integer. Let $\mathcal{F}_{g}$ be a list of global function fields over $k$ of genus $g$. (In this report we will only consider the case of $\mathcal{F}_{2}$, the fields of genus 2.) For each function field $F \in \mathcal{F}_{g}$, do the following:

- Compute the class group $\mathrm{Cl}(F)$ of $F$.

- For each rational place $O$ compute the image $I_{O}$ of the rational places in $\mathrm{Cl}(F)$ under the map $P \mapsto[P-\operatorname{deg}(P) O]: \mathbf{P}_{F} \rightarrow \mathrm{Cl}(F)$.

- List all subgroups of $\mathrm{Cl}(F)$. For each of these, $G$, compute its index $d_{G}$ and the cardinality $m_{G, O}$ of its intersection with $I_{O}$. This gives the existence of an unramified abelian extension of $F$ of degree $d_{G}$, of genus $d_{G}(g-1)+1$ and with $d_{G} m_{G, O}$ rational places.

\section{IMPLEMENTATION AND RESULTS}

Using Magma [1] we implemented the search with all genus 2 curves as base, over $k=\mathbf{F}_{q}$ for $q \leq 16$. For $q$ smaller than 9 the listing of genus 2 curves could be done in the straightforward way; for the bigger values of $q$ we used the Magma package g2twist [5]. The curves are then represented by Magma as hyperelliptic curves. For each such curve we computed its Jacobian and a list of its subgroups, using the arithmetic geometry part of Magma. All the records we found were then checked using the function field part of Magma, this is also how we describe them in this report.

Below we have recorded all the new curves we found by this method, together with the details required for their construction: First a genus 2 function field $F$. Then a set of rational places $M$ that generate the subgroup of the class group that 
defines the extension. More precisely, the extension is $F_{O}^{G}$ where $O$ is any place in $M$ and $G$ is the subgroup of $\mathrm{Cl}(F)$ generated by $\{[P-O]\}_{P \in M}$. (It can also be constructed as the $S$-Hilbert class field of $F$, with $S=\mathbf{P}_{F} \backslash M$.)

The genus 2 fields are always given as a degree 2 extension $F=k(x, y) / k(x)$ defined by some $f \in k[x][y]$. We use the following notation for the rational places of $F$ : For $\alpha \in k$ let $Q_{\alpha}$ be the place of $k(x)$ containing $x-\alpha$. If $Q_{\alpha}$ ramifies then there is a unique place of $F$ containing $x-\alpha$ which we denote by $P_{\alpha}$. If $Q_{\alpha}$ splits there are two such places, one for each $\beta \in k$ such that $f(\alpha, \beta)=0$; it is characterized by containing $y-\beta$, we denote it $P_{\alpha, \beta}$. If there is a unique place of $F$ containing $1 / x$ we denote it $P_{\infty}$.

Curves in characteristic 2. This is the case where most of the work of constructing curves has been done, in particular using the present method, and for $q=2,4$ and 8 we didn't find any improvements.

Over $\mathbf{F}_{16}$ there are 8470 different genus 2 curves. Among their unramified abelian covers we found 2 new records:

- $g=8, N=63 . F: y^{2}+\left(x^{2}+x\right) y=\alpha^{6} x^{5}+\alpha^{12} x^{4}+x^{3}+\alpha^{3} x^{2}+\alpha^{9} x$, where $\alpha$ is a primitive element of $\mathbf{F}_{16}$ satisfying $\alpha^{4}+\alpha+1=0$. Places: three ramified, $P_{\infty}, P_{0}, P_{1}$ and the 3 pairs $P_{\alpha^{3}, \alpha^{4}}, P_{\alpha^{3}, \alpha^{10}}, P_{\alpha^{6}, \alpha^{6}}, P_{\alpha^{6}, \alpha^{12}}$ and $P_{\alpha^{9}, \alpha^{3}}, P_{\alpha^{9}, \alpha^{9}}$. Old interval $[62,75]$.

- $g=49, N=240 . F: y^{2}+\left(x^{2}+x\right) y=x^{5}+x^{3}+x^{2}+x$. Places: With $O$ any of $P_{\infty}, P_{0}, P_{1}$, there are two such extensions. One in which these three places and $P_{\alpha^{5}, \alpha^{2}}$ and $P_{\alpha^{5}, \alpha^{8}}$ splits; and one in which these three places and $P_{\alpha^{10}, \alpha}$ and $P_{\alpha^{10}, \alpha^{4}}$ splits. Old interval $[213,286]$.

Curves in characteristic 3. For $q=3$ we didn't find any new curves. For $q=9$ there had been much previous activity, e.g., [2], 3], 7], [14. By doing a systematic search we found 3 small improvements of the old table entries. Let $\alpha$ be a primitive element of $\mathbf{F}_{9}$ satisfying $\alpha^{2}-\alpha-1=0$.

- $g=18, N=68 . F: y^{2}=x^{5}+\alpha^{6} x^{3}+\alpha^{6} x^{2}+\alpha^{3} x$. Places: $P_{\infty}, P_{0}, P_{1}$ and P. Old interval $[67,84]$.

- $g=32, N=93 . F: y^{2}=x^{5}+\alpha^{6} x^{4}+\alpha^{7} x^{3}+2 x^{2}+\alpha^{5} x+\alpha^{2}$. Places: $P_{\infty}$,

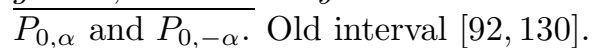

- $g=38, N=$ 111. $F: y^{2}=x^{5}+\alpha^{7} x^{3}+2 x^{2}+\alpha^{2} x$. Places: $P_{\infty}, P_{0}$ and $P_{-\alpha}$. Old interval [105, 149].

Curves over $\mathbf{F}_{5}$. For $q=5$ there had been some previous work, e.g., [8, 9] and [10, in particular using the present method. By doing the systematic search we found 3 improvements of the old table entries:

- $g=7, N=24 . F: y^{2}=x^{5}+x^{2}-x$. Places: $P_{\infty}, P_{0}, P_{4,1}$ and $P_{4,4}$. Old interval $[22,26]$.

- $g=9, N=32 . F: y^{2}=x^{5}-x^{3}+x$. Places: $P_{\infty}, P_{0}$ and either the two places containing $x-1$ or the two places containing $x-4$. Old interval $[26,32]$.

- $\underline{g=12, N=33 .} F: y^{2}=x^{5}+x^{4}+3 x^{2}+1$. Places: $P_{\infty}, P_{0,1}, P_{0,4}$. Old interval [30,38].

We give some details about the construction of the genus 9 curve that we found which, together with the Oesterlé bound, show that $\mathrm{N}_{5}(9)=32$.

Example. Let $k=\mathbf{F}_{5}$ and let $F / k$ be the hyperelliptic field of genus 2 given by

$$
y^{2}=x^{5}-x^{3}+x .
$$

Take $O$ to be $P_{0}$, the unique place containing $x$. Magma gives an isomorphism from $\mathrm{Cl}(F)$ to $\mathbf{Z} / 8 \oplus \mathbf{Z} / 8$ under which the divisor classes $\left[P_{\infty}-O\right],\left[P_{4,3}-O\right]$ and 
$\left[P_{4,2}-O\right]$ map to $(0,4),(0,5)$ and $(0,3)$. They are hence contained in a subgroup of index 8. Therefore, $F$ has an unramified extension of degree 8 in which these places and $O$ split completely. This extension has genus $1+8 \cdot(2-1)=9$ and $8 \cdot 4=32$ rational places.

We mention that Magma can give explicit equations for the class fields: The degree 8 extension of $F$ in this example has minimal polynomial

$$
\begin{aligned}
T^{8} & +\left((4 x+4) y+3 x^{4}+3 x^{3}+3 x^{2}+3 x+3\right) T^{4}+ \\
& +\left(x^{5}+2 x^{4}+2 x^{3}+2 x^{2}+2 x+1\right) y+x^{8}+2 x^{6}+x^{5}+2 x^{4}+x^{3}+2 x^{2}+1 .
\end{aligned}
$$

Curves over $\mathbf{F}_{7}, \mathbf{F}_{11}$ and $\mathbf{F}_{13}$. For $q=7,11$ and 13 there where no lower bounds in the tables for genus greater than 4 , so all we had to do to make it to the tables was to produce curves with more than $b(g) / \sqrt{2}$ points, where $b(g)$ is the best known upper bound for $\mathrm{N}_{q}(g)$. (This is the criteria used to decide if a curve is considered to have many points, see [4.) The results are given in the table below; we give integers $g$ and $N$ such that there exists a genus $g$ curve with $N$ points, and then the interval $[b(g) / \sqrt{2}, b(g)]$.

The details of the construction are given in 12 and [13. There, for each entry given in this table, a function field of genus 2 together with all the rest of the information required to prove the existence of the the extension, are given.

New entries for the tables over $\mathbf{F}_{7}, \mathbf{F}_{11}$ and $\mathbf{F}_{13}$.

\begin{tabular}{|l|l|l||l|l|l||l|l|l|}
\hline$q=7$ & & & $q=11$ & & & $q=13$ & & \\
\hline$g$ & $N$ & {$\left[\frac{b(g)}{\sqrt{2}}, b(g)\right]$} & $g$ & $N$ & {$\left[\frac{b(g)}{\sqrt{2}}, b(g)\right]$} & $g$ & $N$ & {$\left[\frac{b(g)}{\sqrt{2}}, b(g)\right]$} \\
\hline 5 & 24 & {$[20,28]$} & 5 & 36 & {$[27,38]$} & 5 & 40 & {$[32,44]$} \\
6 & 25 & {$[23,32]$} & 6 & 40 & {$[32,45]$} & 6 & 50 & {$[37,52]$} \\
7 & 30 & {$[26,36]$} & 7 & 42 & {$[36,50]$} & 7 & 48 & {$[42,58]$} \\
8 & 35 & {$[27,38]$} & 8 & 42 & {$[39,55]$} & 8 & 56 & {$[45,63]$} \\
9 & 32 & {$[29,41]$} & 9 & 48 & {$[42,59]$} & 9 & 56 & {$[49,68]$} \\
10 & 36 & {$[32,45]$} & 10 & 54 & {$[45,63]$} & 10 & 63 & {$[51,72]$} \\
11 & 40 & {$[34,48]$} & 11 & 60 & {$[48,67]$} & 11 & 70 & {$[55,77]$} \\
12 & 44 & {$[37,51]$} & 12 & 66 & {$[51,72]$} & 12 & 66 & {$[58,82]$} \\
13 & 48 & {$[39,54]$} & 13 & 60 & {$[55,77]$} & 13 & 72 & {$[62,87]$} \\
14 & & & 14 & & & 14 & 65 & {$[65,91]$} \\
15 & & & 15 & 70 & {$[61,85]$} & 15 & 84 & {$[68,96]$} \\
16 & 45 & {$[45,63]$} & 16 & & & 16 & 90 & {$[72,101]$} \\
17 & 64 & {$[47,66]$} & 17 & 80 & {$[66,93]$} & 17 & 96 & {$[75,105]$} \\
18 & 51 & {$[49,68]$} & 18 & 85 & {$[70,98]$} & 18 & 85 & {$[78,110]$} \\
19 & 54 & {$[51,71]$} & 19 & 90 & {$[73,102]$} & 19 & 90 & {$[82,115]$} \\
20 & & & 20 & 76 & {$[75,106]$} & 20 & 95 & {$[85,119]$} \\
21 & 60 & {$[55,77]$} & 21 & 80 & {$[78,110]$} & 21 & 100 & {$[88,124]$} \\
22 & 63 & {$[56,79]$} & 22 & & & 22 & 105 & {$[92,129]$} \\
23 & 66 & {$[58,82]$} & 23 & 88 & {$[85,119]$} & $\ldots$ & & \\
24 & & & 24 & 92 & {$[87,123]$} & 29 & 140 & {$[114,161]$} \\
25 & 72 & {$[62,87]$} & 25 & 96 & {$[90,127]$} & 32 & 124 & {$[124,175]$} \\
26 & & & 26 & & & 33 & 128 & {$[127,179]$} \\
27 & 78 & {$[66,93]$} & 27 & & & 35 & 136 & {$[133,187]$} \\
& & & 28 & 108 & {$[98,138]$} & 36 & 140 & {$[136,191]$} \\
& & & $\ldots$ & & & 37 & 144 & {$[138,195]$} \\
& & & 33 & 128 & {$[111,156]$} & 41 & 160 & {$[150,211]$} \\
& & & & & 43 & 168 & {$[155,219]$} \\
\hline
\end{tabular}


Acknowledgments. The author thanks the Wenner-Gren Foundations for financial support; the Korteweg-de Vries Institute at the University of Amsterdam for hospitality; and Gerard van der Geer for his hospitality and for helpful conversations and comments.

\section{REFERENCES}

[1] W. Bosma, J. Cannon, C. Playoust, The Magma algebra system I: The user language, J. Symbolic Comput., 24 (1997), 235-265.

[2] G. van der Geer, Hunting for curves with many points, In: C. Xing et al. (Eds.): IWCC 2009, LNCS 5557, pp. 82-96, 2009. Available electronically: arXiv:0902.3882 (2009)

[3] G. van der Geer, M. van der Vlugt How to construct curves over finite fields with many points In: Arithmetic Geometry, (Cortona 1994), F. Catanese Ed., Cambridge Univ. Press, Cambridge, 1997, p. 169-189.

[4] G. van der Geer and M. van der Vlugt, Tables of curves with many points, Math. Comp 69, (2000), no 230 pp. $797-810$

[5] R. Lercier and C. Ritzenthaler, G2Twists v1.1: Reconstruction of genus 2 curves from their invariants and twists over a finite field, Magma package, incorporated in the official magma distribution, since version 2.15, April 2009.

[6] manYPoints.org, Tables of upper and lower bounds for the maximum number of points on a genus $g$ curve over different finite fields. Moderators: G. van der Geer, E. Howe, K. Lauter, C. Ritzenthaler

[7] H. Niederreiter; C. P. Xing A general method of constructing global function fields with many rational places In: Algorithmic Number Theory (Portland 1998), Lecture Notes in Comp. Science 1423, Springer, Berlin, 1998, p. 555-566.

[8] H. Niederreiter; C. P. Xing, Cyclotomic function fields, Hilbert class fields and global function fields with many rational places Acta Arithm. 79 (1997), p. 59-76.

[9] H. Niederreiter; C. P. Xing, Global function fields with many rational places over the quinary field Demonstratio Math. 30 (1997), no. 4, 919-930

[10] H. Niederreiter; C. P. Xing, Global function fields with many rational places over the quinary field. II Acta Arith. 86 (1998), no. 3, 277-288

[11] H. Niederreiter; C. P. Xing, Rational Points on Curves over Finite Fields, LMS Lecture Note Series 285, 2001

[12] K. Rökaeus, Computer search for curves with many points among unramified covers of genus 2 curves over $\mathbf{F}_{5}, \mathbf{F}_{7}, \mathbf{F}_{9}$ and $\mathbf{F}_{11}$ Report, available under manYPoints.org, http://manypoints.org/upload/1369548137.pdf

[13] K. Rökaeus, Computer search for curves with many points among abelian covers of genus 2 curves over $\mathbf{F}_{13}$ Report, available under manYPoints.org, http://manypoints.org/upload/1225383491.pdf

[14] V. Shabat, Curves with many points, Thesis, University of Amsterdam, 2001.

[15] H. Stichtenoth, Algebraic function fields and codes, Graduate Texts in Mathematics 254, Springer-Verlag, 2009

Karl Rökaeus, Korteweg de Vries Instituut voor Wiskunde, Universiteit van Amsterdam, P.O. Box 94248, 1090 GE Amsterdam, The Netherlands

E-mail address: S.K.F.Rokaeus@uva.nl 